\title{
On the Nature of the Chemical Noise in MALDI Mass Spectra
}

\author{
Andrew N. Krutchinsky and Brian T. Chait* \\ Rockefeller University, New York, New York, USA
}

The so-called "chemical noise background" imposes a major limit on the practical sensitivity of MALDI mass spectrometry. Typically, as the amount of material of interest subjected to MALDI analysis is reduced, the signal decreases to the point where it can no longer be differentiated from the chemical noise. Using a newly designed MALDI-ion trap mass spectrometer, we describe experiments intended to throw light on the nature of the chemical noise background and to reduce its effects. Single-stage mass spectrometric signals from peptides were observed to disappear into the noise when the amount of sample applied to the MALDI sample stage was decreased to less than a femtomole. At these low levels, analysis of the collision-induced fragmentation spectra revealed the presence of ions originating from the peptide as well as cluster ions that originate from the chemical noise. The fragmentation pattern arising from dissociation of the cluster species suggests that they are composed largely of matrix molecules. A significant fraction of these cluster ions can be dissociated at activation energies lower than the threshold for peptide fragmentation. We used this finding to collisionally pre-activate MALDI ions to remove a significant portion of the chemical noise from the spectrum, allowing us to obtain readily discernible single stage MS signals from 100 attomols of peptide. The strategy also yielded high quality MS/MS spectra from 100 attomols of peptide. Different possibilities of collisional pre-activation for improving sensitivity are considered. (J Am Soc Mass Spectrom 2002, 13, 129-134) @ 2002 American Society for Mass Spectrometry

Developments in mass spectrometry (MS) technology over the past two decades have led to astonishing improvements in the sensitivity for peptide analysis [1-3]. Key to this success has been the introduction and refinement of matrix-assisted laser desorption/ionization (MALDI) and electrospray ionization (ESI), enhancements of the efficiency and performance of mass analyzers, improvements in mass spectral analysis tools, as well as optimization of sample handling techniques. These developments have advanced MS to the level of a tool of choice for protein identification and characterization.

Currently, the practical limit in sensitivity is usually imposed by background ions in the mass spectrum rather than the inherent sensitivity of the mass spectrometer. Inspection of mass spectra obtained in our MALDI/ESI-QqTOF [4] and MALDI/ESI-ion trap instruments [5] reveal background ion peaks at essentially every $\mathrm{m} / \mathrm{z}$ value. Close examination of these individual background peaks at higher resolutions indicates the presence of a large number of different ion species at each $\mathrm{m} / \mathrm{z}$ value (data not shown). Although the presence of this "chemical noise" is widely recognized and numerous attempts

Published online November 30, 2001

Address reprint requests to Brian T. Chait, Rockefeller University, 1230 York Avenue, New York, NY 10021. E-mail: chait@rockvax.rockefeller.edu have been made to reduce its effect [6-12], we do not yet have a detailed understanding of its nature and origins.

When the magnitude of the signal from analyte ions becomes comparable to that of background ions, the ion peaks of interest begin to merge with the noise and can no longer be distinguished unless special accommodations are made to increase the signal-to-noise ratio. Thus, for example, we can accumulate the spectra for longer times. However, the resulting statistical improvement of the signal-to-noise increases rather slowly with accumulation time, $\mathrm{t}$ (i.e., $\propto \mathrm{t}^{0.5}$ ). This increased time for MS analysis leads to premature consumption of the sample before the full analysis can be completed (especially for multiple MS/MS measurements of the components of peptide mixtures). Another approach for overcoming the noise limitation utilizes linked scan modes of operation [13]. Unfortunately, current realizations of this technique have efficiencies that are compromised by the low duty cycle incurred by the need to scan the mass spectrometer. Selective MS/MS analysis of the ions of interest can greatly improve the duty cycle; however it introduces a problematic situation in that we may not know a priori the $\mathrm{m} / \mathrm{z}$ values of the species to be fragmented.

In the present paper we report results on a study of factors that limit sensitivity in a newly constructed 


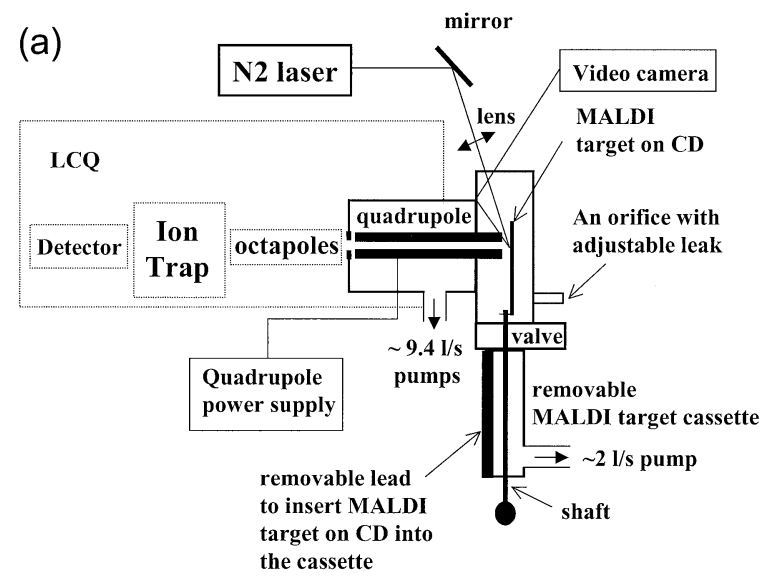

(b)



Figure 1. (a) Schematic diagram of the MALDI-ion trap mass spectrometer. (b) Detailed diagram of the quadrupole ion guide construction and voltages applied to the different components of the MALDI ion interface.

MALDI-IT mass spectrometer [5]. Our findings provide information on the origin of the chemical noise in MALDI spectra. We use this information to demonstrate and suggest methods for improving sensitivity.

\section{Experimental}

\section{MALDI-Ion Trap Mass Spectrometer}

We recently modified a commercial ion trap (IT) mass spectrometer (Thermo Finnigan LCQ Classic, San Jose, CA) and installed a new ion interface, which enabled high performance operation in both MALDI and/or ESI modes [5]. Here we describe results obtained with a version of the instrument that operates exclusively in the MALDI mode.

Figure 1 shows a schematic diagram of the mass spectrometer, where the added MALDI ion source and new interface is shown with the solid lines (Figure 1a). The interface consists of a quadrupole $(\sim 20 \mathrm{~cm}$ long, $0.635 \mathrm{~cm}$ rod diameter) installed between the octapoles and the skimmer in the original commercial configuration. The quadrupole is separated from the octapoles by an aperture plate (aperture diameter $\sim 0.3 \mathrm{~cm}$ ). Figure $1 \mathrm{~b}$ provides a detailed view of the quadrupole construction and the voltages applied to the different compo- nents. The additional quadrupole acts as a high pressure ion guide [14-16]. The quadrupole is driven by an independent RF power supply, which consists of a 500 $\mathrm{kHz}$ crystal oscillator-controlled sine wave generator and a power amplifier (model 240L ENI, Rochester, $\mathrm{NY}$ ), which produces an RF voltage with the typical value of the sine wave amplitude $\mathrm{A}=+[300-500] \mathrm{V}$. More details on the circuitry can be found elsewhere [4, $5,16]$.

The ion guide assembly also contains an accelerator, which provides an electrical force to drag the ions towards the exit of the ion guide. The accelerator consists of a set of four $\sim 18 \mathrm{~cm}$ long rods $0.32 \mathrm{~cm}$ in diameter inserted between the main rods of a quadrupole ion guide (see the assembly cross section in Figure $1 b)$. The accelerator rods are closer to the axis of the quadrupole ion guide at its entrance and further from the axis at the ion guide output. A constant voltage (typically $+100 \mathrm{~V}$ ) applied to all four rods of the accelerator creates a small electrical field along the axis of the quadrupole ion guide because of the changing proximity of these rods to the axis of the ion guide. Its presence in our construction improves the transport efficiency of ions [5].

The pressure in the quadrupole ion guide of $70 \pm 10$ mTorr is controlled by the ratio of the rate of flow of gas introduced from a small orifice with an ambient air adjustable leak (see Figure 1a) and the pumping speed of the mechanical pump (Pfeifer UNO 030B, $\sim 81 / \mathrm{s}$ ) originally installed in the instrument. This pump also evacuates the turbomolecular pump supplied with the original instrument, which maintains the pressure in the detector region at $\sim 2 * 10-5$ Torr.

MALDI samples are deposited on the polycarbonate surface of a compact disc (CD), which serves as a MALDI target. We have reported the major characteristics of the polycarbonate MALDI target elsewhere [5].

The added section (Figure 1a) has a sample inlet system that allows rapid (1-2 $\mathrm{min}$ ) introduction of the CD MALDI target through a vacuum lock. The $C D$ with samples spotted on its surface is fixed with a screw to a metal CD support plate and introduced into the mass spectrometer. A MALDI target potential is applied to the plate when it makes a physical contact with a spring electrode. The distance between the CD and the entrance of the ion guide is $\sim 1 \mathrm{~mm}$. Each sample on the $\mathrm{CD}$ is positioned at the entrance of the quadrupole ion guide by rotating and translating a shaft attached to the CD supporting plate. Rotation of the shaft is transmitted to the CD through a small rubber wheel at the end of the shaft.

A $337 \mathrm{~nm}$ wavelength laser beam (VSL-337 nitrogen laser, Laser Science Inc., Franklin, MA) operating at a repetition rate of $10-20 \mathrm{~Hz}$ is reflected by a mirror and introduced through a collimating lens $(\mathrm{f}=15 \mathrm{~cm})$ and then through a sapphire window to the surface of the $\mathrm{CD}$ at an angle of incidence of $\sim 60^{\circ}$. The apparent diameter of the laser spot on the sample surface as measured by burning a spot on a sensitive surface is $\sim 0.3-0.5 \mathrm{~mm}$. The power density of laser radiation in 
the spot is (2-5) $\times 10^{7} \mathrm{~W} / \mathrm{cm}^{2}$, controlled by a variable attenuator (Model 935-5, Newport Corp., Irvine, CA). Both the sample and the laser spot are monitored by a video camera. Desorbed ions are introduced directly into the quadrupole ion guide.

\section{Ion Trap Settings}

All spectra were obtained with the following instrument settings (using the standard Finnigan notation): ion injection time $500 \mathrm{~ms}$ ("Maximum Inject Time"), automatic gain control (AGC) on, maximum number of ions allowed to fill the trap $5^{*} 10^{9}$ ("Full MS Target"). In addition, MS/MS spectra were obtained with settings - $m / z$ window was 3-4 ("Isolation Width"), activation energy was $25 \%$ ("Normalized Collision Energy"), q of activation was 0.25 ("Activation q") and activation time was $300 \mathrm{~ms}$ ("Activation Time").

\section{Sample Preparation}

A calibration stock mixture consisting of six peptides (bradykinin fragment 2-9, Substance $P$, neurotensin, amyloid $\beta$-protein fragment 12-28, ACTH fragment 1-24, insulin chain B, oxidized, from bovine insulin) was prepared at a concentration $200 \mathrm{fmol} / \mu \mathrm{l}$ per component in water/ methanol/acetic acid (60/35/5 v/v/v). Dilutions of the stock solution were made just prior to measurements.

Fresh saturated solutions of 2,5--Dihydrobenzoic acid (DHB, MW = 154.1 Da), $\alpha$-Cyano-4-hydroxycinnamic acid (4HCCA, MW = 189.2 Da) and 3,5-dimethoxy 4-hydroxycinnamic acid $(\mathrm{SA}, \mathrm{MW}=224.2 \mathrm{Da})$ matrices (Sigma-Aldrich, Milwaukee, WI) were prepared in $\mathrm{MeOH} / \mathrm{H}_{2} \mathrm{O} /$ Acetic Acid $(60 / 35 / 5 \mathrm{v} / \mathrm{v} / \mathrm{v})$ just prior to measurements. To prepare samples for MS analysis, a volume of the diluted peptide mixture was mixed with an equal volume of the saturated DHB solution and $1 \mu \mathrm{l}$ of the resulting solution was spotted on the CD sample plate.

\section{Results and Discussion}

The present MALDI-ion trap mass spectrometer produces high quality MS and MS/MS spectra from low femtomole amounts of peptide mixtures. An important feature of the new instrument is its ability to perform high speed analyses [5]. Figure 2a shows an example of a MALDI-ion trap mass spectrum obtained from a six peptide mixture, where $1 \mathrm{fmol}$ of each component was deposited on the MALDI target. The spectrum was acquired in $2 \mathrm{sec}$. Signals from five of the six components can be readily discerned above the noise. The bottom panel of the same figure shows the MS/MS spectrum of the single peptide component at $m / z$ 1673. A useful signal-to-noise ratio was obtained in a 5-second acquisition. However, when we further diluted the sample by factor of 10 and applied the resulting $0.1 \mathrm{fmol}$ of the peptide mixture to the MALDI target, we were no longer able to
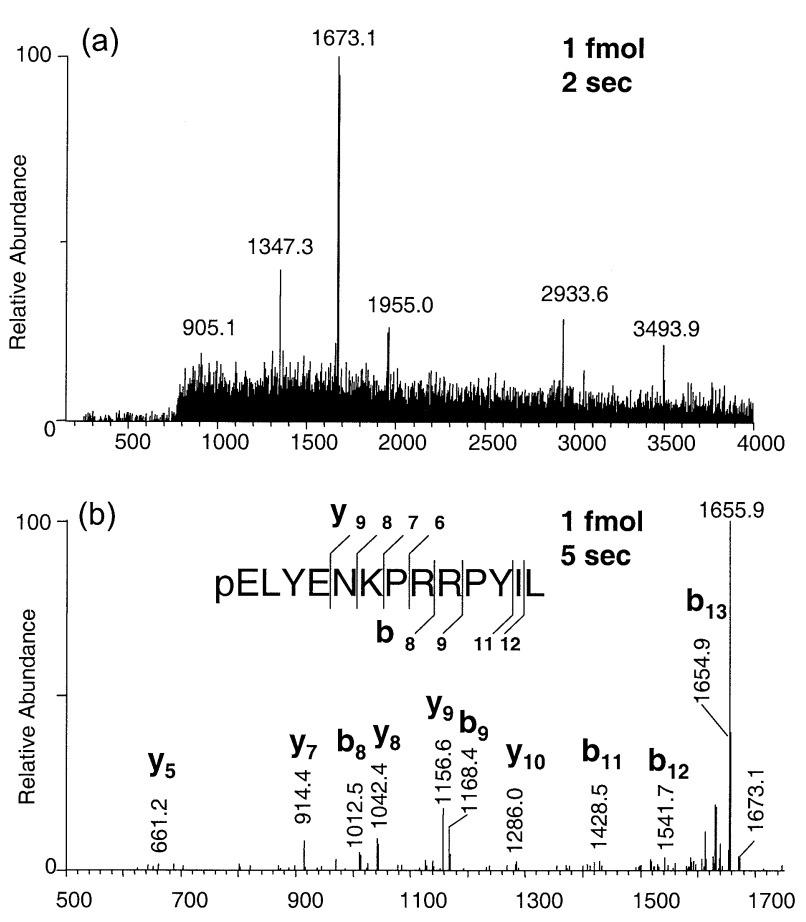

Figure 2. (a) MALDI mass spectrum of $1 \mathrm{fmol}$ each of a mixture of six peptides, bradykinin fragment 2-9 (monoisotopic mass 903.5 $\mathrm{Da})$, substance P (1346.7 Da), neurotensin (1671.9 Da), amyloid $\beta$-protein fragment 12-28 (1954.0 Da), ACTH fragment 1-24 (2931.6 $\mathrm{Da})$, oxidized bovine insulin chain B (3493.6 Da). Spectrum acquisition time was 2 sec. (b) MALDI-MS/MS spectrum of neurotensin with the corresponding observed fragments indicated. Spectrum acquisition time was $5 \mathrm{sec}$.

discern the peptide ion signals even when the acquisition time was increased to $1 \mathrm{~min}$ (Figure 3a). The persistent signal from the background ions interferes with the observation of the ion signals from the peptides. This is a typical situation that is frequently encountered in the analysis of small amounts of sample. The detection limit varies from instrument to instrument, but in general depends on sample handling and preparation procedures as well as on particular characteristics of the mass spectrometer, including for example the resolution and the type of ion detector used.

Despite the absence of discernible peptide ion signals at $0.1 \mathrm{fmol}$ in the single-stage mass spectrum, we were able to obtain a diagnostic MS/MS spectrum of the species at $\mathrm{m} / \mathrm{z} 1673$ using an acquisition period of $1 \mathrm{~min}$ (Figure $3 b$ ). Although the MS/MS spectrum is noisy, fragments of the peptide can be clearly observed in the spectrum (compare Figures $2 \mathrm{~b}$ and $3 \mathrm{~b}$ ). These diagnostic fragments arise from selective fragmentation in the ion trap $[17,18]$ of the singly charged peptide ions at a few characteristic places along the peptide backbone, namely on the $\mathrm{N}$-terminal side of proline residues $\left(b_{9}\right.$, $\left.\mathrm{y}_{7}\right)$ and the C-terminal side of the glutamic acid residue $\left(\mathrm{y}_{9}\right)$. Another striking feature of the MS/MS spectrum is a series of fragments separated by $\sim 154 \mathrm{Da}$ and $\sim 136$ Da starting from the parent ion. We hypothesized that this series arises from the fragmentation of clusters 

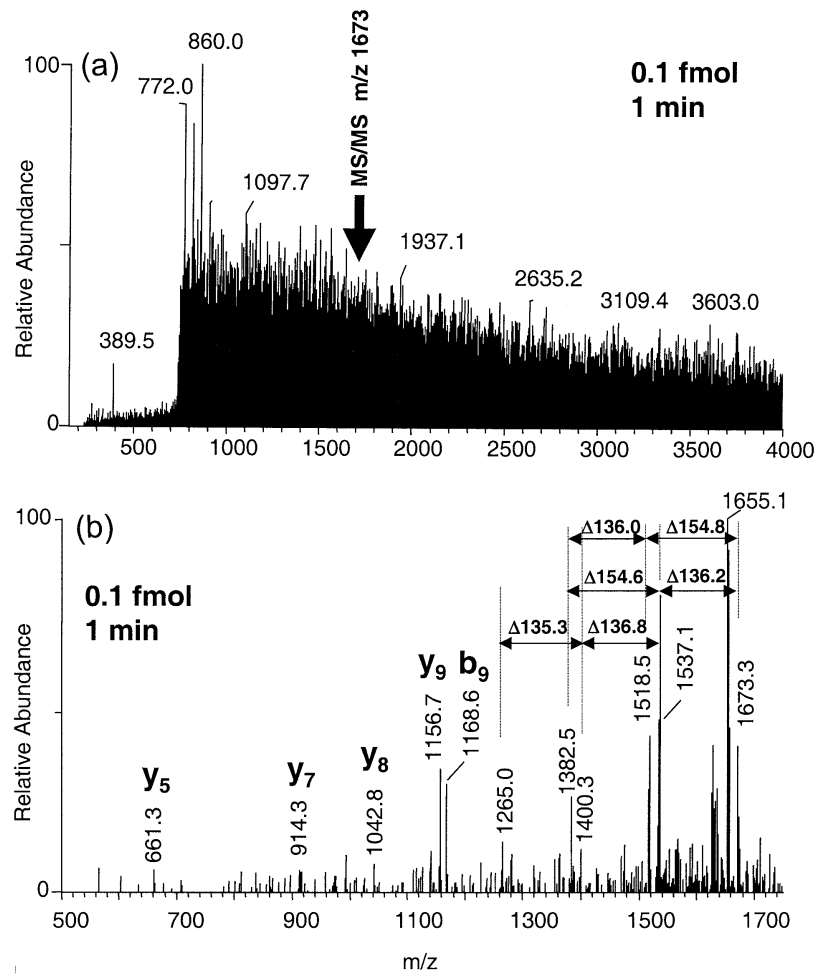

Figure 3. (a) MALDI mass spectrum of the same six peptide mixture described in the legend to Figure 2. The amount of each peptide applied to the $\mathrm{CD}$ target was $0.1 \mathrm{fmol}$. Acquisition time was 1 min. (b) MALDI-MS/MS mass spectrum of the species at $\mathrm{m} / \mathrm{z}$ 1673. The observed fragments corresponding to neurotensin (see sequence in Figure 2) are indicated.

containing intact and fragmented matrix molecules formed during the MALDI plume expansion and ionization process. The molecular mass of DHB is $154.1 \mathrm{Da}$, while elimination of a water molecule from DHB would yield an entity of molecular mass $136.1 \mathrm{Da}$. Thus, the spectrum in Figure $4 \mathrm{~b}$ exhibits a series of characteristic losses that is consistent with successive loss of either intact DHB molecules or its fragments from the parent ion species selected at $m / z 1673$. Supporting evidence in favor of this hypothesis was the observation of essentially the same set of characteristic losses in the MS/MS spectra at every $\mathrm{m} / \mathrm{z}$ value tested. Figure 4 shows a representative subset of such MS/MS spectra. We conclude that these cluster ions have a composition $(\mathrm{DHB})_{\mathrm{n}} \mathrm{XC}^{+}$, where $\mathrm{X}$ represents presently unknown species and $\mathrm{C}^{+}$represents a cation.

We were unable to determine the exact composition of these cluster species via $\mathrm{MS}^{\mathrm{n}}$ experiments because of the increasing number of dissociation channels that open up as a function of the order of $\mathrm{MS}^{\mathrm{n}}$ experiment, the low absolute intensity of the chemical noise produced from our MALDI samples on the polycarbonate CD surface, and the complex mix of ion species that are present at each nominal $\mathrm{m} / \mathrm{z}$ value. We also investigated the use of several alternative MALDI matrices including $\alpha$-cyano-4-hydroxycinnamic acid (4HCCA) and 3,5-dimethoxy 4-hydroxycinnamic acid (SA). MALDI mass
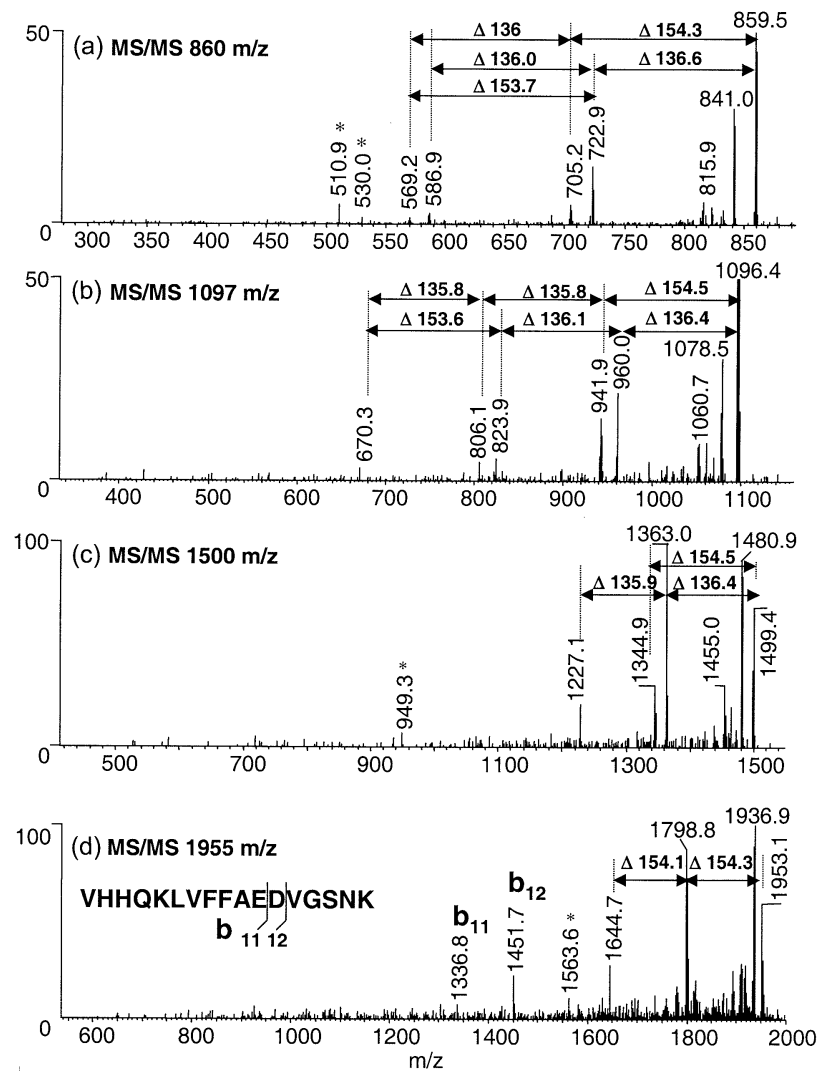

Figure 4. MALDI-MS/MS mass spectra obtained from a selection of $m / z$ values in Figure 3a. These include $m / z$ values $(860,1097$, 1500) where no peptide signals are expected as well as one $\mathrm{m} / \mathrm{z}$ value (1955) where a peptide signal is expected. Characteristic losses of intact molecules of DHB (154 Da) and molecules of DHB with eliminated water $(136 \mathrm{Da})$ from the parent species are indicated. The asterisk indicates unexplained ion peaks.

spectra of the six peptide mixture demonstrated that these matrices were inferior to DHB in conditions optimized for our MALDI-IT mass spectrometer in that the peptide ion signals were less intense, the shot-toshot reproducibility was lower, and the noise level higher. The likely reason for this inferior behavior is the "hotter" nature of 4HCCA and SA compared with DHB [19]. However, the spectra from 4HCCA and SA also showed characteristic losses evidencing the cluster nature of the background from these matrices as well (data not shown).

Our finding that DHB clusters readily undergo collision-induced dissociation can be used to improve the likelihood of observing weak ion signals in the presence of the chemical noise. The technique is demonstrated in Figure 5 where we carried out "broadband" collisional activation of part of the mass spectrum in Figure 3a centered on $\mathrm{m} / \mathrm{z}$ 1673. Activation was performed on ions isolated in a window with width $\pm 50 \mathrm{~m} / \mathrm{z}$ units. The top panel shows this selection window with no activation applied to the selected ions. Under these conditions, we observe the same chemical noise background in the selected portion of the spectrum as observed in Figure 3a, with no 


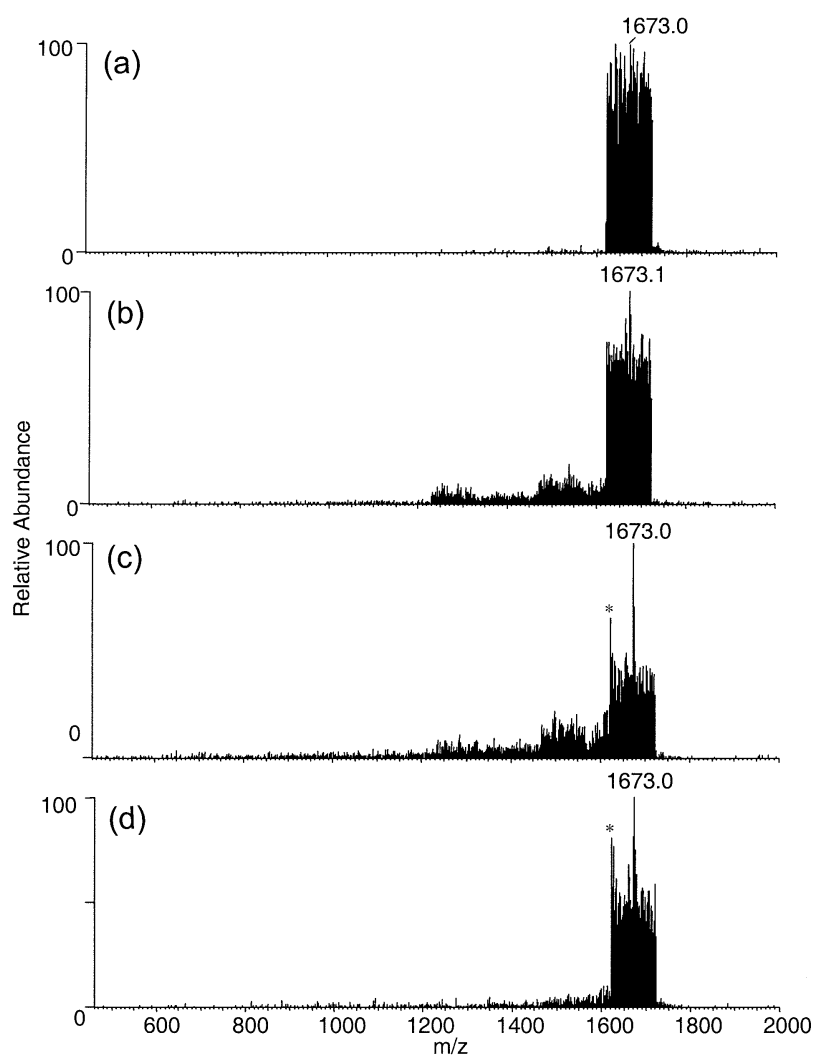

Figure 5. MALDI spectra of the species from the $0.1 \mathrm{fmol}$ peptide mixture obtained in $100 \mathrm{~m} / \mathrm{z}$ unit wide window centered on $\mathrm{m} / \mathrm{z}$ 1673 with (a) no collisional activation applied to the selected ions in the window; (b) mild collisional activation of the species in the selected window (i.e., performed at a "normalized collision energy" of $15 \%$ for a period of $300 \mathrm{~ms}$ ); (c) stronger collisional activation of the species in the selected window (i.e., performed at a "normalized collision energy" of $20 \%$ for a period of $300 \mathrm{~ms}$ ). (d) shows the result of a double collisional activation experiment. Here, the selected species were activated at $20 \%$ "normalized collision energy" for $300 \mathrm{~ms}$. The asterisk designates an artifact "peak" that we have observed to appear at the edge of the selection window.

discernible peptide signal. However, after modest activation of the ion species in the selected window, we observe dissociation of ions within the window, and the peptide ion at $m / z 1673$ begins to emerge from the noise (Figure $5 b$ ). It is of note to observe that the center of the distribution arising from fragmentation of the species in the selected window is shifted by $\sim 150 \mathrm{~m} / z$ units, which likely corresponds to the loss of a single DHB molecule from each of the cluster species in the window. When we further increase the activation energy to the threshold for fragmentation of the peptide ion, the peptide ion peak becomes clearly apparent (Figure 5c). Further increases of the excitation did not produce any further improvement in the signal-to-noise ratio in the selected window; but rather increased the fragmentation of the peptide ions at $m / z$ 1673. The lower panel of Figure 5 shows a $\mathrm{MS}^{3}$ experiment. Ions in the selected window were excited under the same conditions used in Figure 5c,

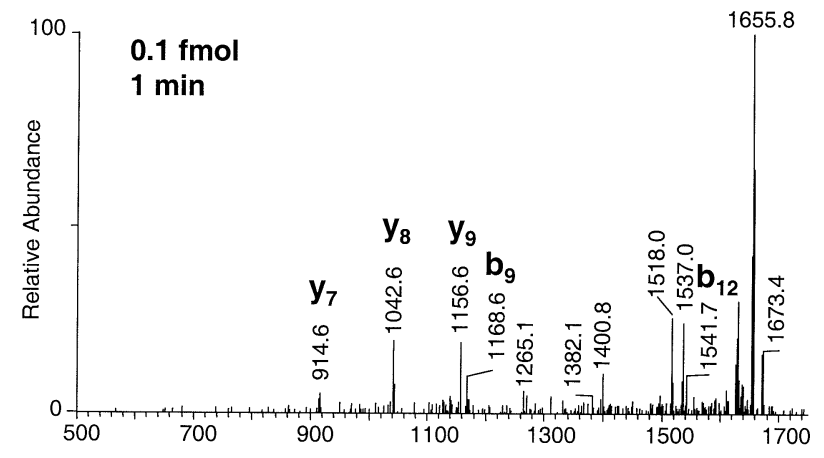

Figure 6. MALDI-MS ${ }^{3}$ spectrum obtained from $0.1 \mathrm{fmol}$ of the peptide at $m / z$ 1673. During the $\mathrm{MS}^{2}$ stage of the experiment, the ions were activated at a "normalized collision energy" of $20 \%$ for $300 \mathrm{~ms}$ to remove the background cluster species that readily undergo fragmentation under these collisional activation conditions. During the subsequent $\mathrm{MS}^{3}$ stage of the experiment, the energy was increased to $25 \%$ to obtain fragmentation of the peptide ions.

whereupon the remaining stable species were reisolated and subjected to a second round of excitation under the same conditions that were used to obtain Figure 5c. Very little further dissociation of the residual background is observed, demonstrating that these remaining background ions are stable to dissociation at excitations close to the threshold for peptide dissociation. Thus, there is a relatively stable component of the chemical noise background that is resistant to dissociation. We conclude from this experiment that collisional activation of ion species in the selected window allow us to remove at least half of the chemical noise background species, resulting in the clear observation of the peptide signal with a signal-to-noise ratio $\sim 3: 1$.

To test the utility of the above-described noise reduction procedure, we again obtained a fragmentation spectrum of the peptide at $\mathrm{m} / \mathrm{z} 1673$ from the $0.1 \mathrm{fmol}$ peptide mixture sample. However, this time we performed an $\mathrm{MS}^{3}$ experiment in which we first activated ions at the same energy as that used to obtain Figure 5c causing dissociation of the more fragile components of the chemical noise background, and then increased the activation energy so as to fragment the peptide. The $\mathrm{MS}^{3}$ fragmentation spectrum of the peptide is shown in Figure 6. As expected, the background fragmentation due to noise in this spectrum is lower than that in the corresponding $\mathrm{MS}^{2}$ spectrum (Figure $3 \mathrm{~b}$ ).

\section{Conclusion}

One of the major obstacles in obtaining useful MALDI-MS and MS/MS spectra from low amounts of peptides is interference from the "chemical noise". This "chemical noise" is produced during the desorption and ionization of peptides, matrix and impurities in the sample. Unwanted impurities can be removed to some extent by common sense methods-e.g., by using highly purified reagents, careful desalting procedures, and clean MALDI substrates. Thus, we have found that the polycar- 
bonate surface used in the fabrication of CDs produces the lowest background in MALDI spectra of the various ( 20) surfaces that we have tested to date [5]. While we continue to search for improved procedures and materials that decrease the "chemical noise" arising from impurities, we believe that it will be very difficult to eliminate this source of background completely.

An even more challenging problem is the removal of the "chemical noise" arising from matrix ions. Analysis of MS/MS spectra obtained from weak peaks and background in the MS spectrum revealed the presence of matrix cluster ions at practically every $\mathrm{m} / \mathrm{z}$ value. These cluster species produce a characteristic fragmentation pattern that arises from sequential loss of intact matrix molecules and matrix fragments. In particular, a significant proportion of the clusters of DHB were found to fragment readily at activation energies lower than that used to fragment peptide ions. This phenomenon can be used to pre-activate and break up those matrix-containing clusters that fragment more readily than peptides. Such activation likely occurs to some extent upon ion injection in the ion trap even prior to ion manipulation in the trap.

Another method to pre-activate ions is to perform MS/MS experiment over a wide $\mathrm{m} / \mathrm{z}$ window. By selecting the pre-activation energy to be lower than the threshold energy for peptide fragmentation, we have demonstrated that we can improve the signal-to-noise in the selected $\mathrm{m} / \mathrm{z}$ window. Current settings in the Finnigan ion trap limit the width of the window over which we can pre-activate ions to $100 \mathrm{~m} / \mathrm{z}$ units. If this window can be effectively extended to a width of 200-500 m/z units, we will be able to apply the optimal algorithms for spectra acquisition that we have described previously [5].

We finally consider the possibility of pre-activation of ions external to the ion trap. This should be readily achievable in the high pressure collisional ion guide [5]. For example, ions can be readily pre-activated during use of the ion guide as a narrow $\mathrm{m} / \mathrm{z}$ range filter, where the selected ions are excited because of proximity to the boundaries of stability [20]. Alternative means for preheating the ions are to inject them at higher energy into the ion guide or excite them with "white noise" applied to the RF drive signal.

These procedures should allow us to recognize peptides at increasingly low levels and to determine their masses for subsequent MS/MS analysis.

\section{Acknowledgments}

This work is supported by the National Institute of Health (Grant RR00862 from the National Center for Research Resources and Grant R33CA89810 from National Cancer Institute) and Merck Genome Research Institute.

\section{References}

1. Gygi, S. P.; Aebersold, R. Mass Spectrometry and Proteomics. Curr. Opin. Chem. Biol. 2000, 4, 489-494.
2. Chalmers, M. J.; Gaskell, S. J. Advances in Mass Spectrometry for Proteome Analysis. Curr. Opin. Biotechnol. 2000, 11, 384390.

3. Papac, D. I.; Shahrokh, Z. Mass Spectrometry Innovations in Drug Discovery and Development. Pharm. Res. 2001, 8, 131-145.

4. Krutchinsky, A. N.; Zhang, W.; Chait, B. T. Rapidly Switchable Matrix-assisted Laser Desorption/Ionization and Electrospray Quadrupole-Time-of-Flight Mass Spectrometry for Protein Identification. J. Am. Soc. Mass Spectrom. 2000, 11, 493-504.

5. Krutchinsky, A. N.; Kalkum, M.; Chait, B. T. Rapid, Automatic Identification of Proteins with a MALDI-quadrupole Ion Trap Mass Spectrometer. Anal. Chem. 2001, 73, 5066-5077.

6. Livadaris, V.; Blais, J.-C.; Tabet, J-C. Formation of Non-specific Protein Cluster Ions in Matrix-assisted Laser Desorption/ ionization; Abundances and Dynamical Aspects. Eur. J. Mass Spectrom. 2000, 6, 409-413.

7. Ramsey, R. S.; Goeringer, D. E.; McLuckey, S. A. Active Chemical Background and Noise Reduction in Capillary Electrophoresis/ion Trap Mass Spectrometry. Anal. Chem. 1993, 65, 3521-3524.

8. (a)Mordehai, A. V.; Henion, J. D. Computer-designed Waveform Technique for Reducing Chemical Noise in Atmospheric-pressure Ionization/ion-trap Mass Spectrometry. Rapid Commun. Mass Spectrom. 1993, 7, 1131-1135.(b)Aebi, B.; Henion, J. The Reduction of Chemical Noise in an Atmospheric Pressure Ionization/ion spray Interface for Mass Spectrometry. Rapid Commun. Mass Spectrom. 1996, 10, 947-951.

9. Voyksner, R. D.; Lee, H. Investigating the Use of an Octupole Ion Guide for Ion Storage and High-pass Filtering to Improve the Quantitative Performance of Electrospray Ionization Mass Spectrometry. Rapid Commun. Mass Spectrom. 1999, 13, 1427-1437.

10. Guevremont, R.; Barnett, D. A.; Purves, R. W.; Vandermey, J. Analysis of a Tryptic Digest of Pig Hemoglobin Using ESIFAIMS-MS. Anal. Chem. 2000, 72, 4577-4584.

11. Karas, M.; Gluckmann, M.; Schafer, J. Ionization in Matrixassisted Laser Desorption/ionization: Singly charged Molecular Ions are the Lucky Survivors. J. Mass Spectrom. 2000, 35, 1-12.

12. Keller, B. O.; Li, L. Discerning Matrix-cluster Peaks in Matrixassisted Laser Desorption/ionization Time-of-flight Mass Spectra of Dilute Peptide Mixtures. J. Am. Soc. Mass Spectrom. 2000, 11, 88-93.

13. Schwartz, J. C.; Wade, A. P.; Enke, C. G.; Cooks, R. G. Systematic Delineation of Scan Modes in Multidimensional Mass Spectrometry. Anal. Chem. 1990, 62, 1809-1818.

14. Douglas, D. J.; French, J. B. Collisional Focusing Effects in Radiofrequency Quadrupoles. J. Am. Soc. Mass Spectrom. 1992, 3, 398-408.

15. Xu, H. J.; Wada, M.; Tanaka, J.; Kawakami, H.; Katayama, I. A New Cooling and Focusing Device for Ion Guide . Nucl. Instrum. Methods Phys. Res., Sect. A 1993, 333(2-3), 274-281.

16. Krutchinsky, A. N.; Chernushevich, I. V.; Spicer, V. L.; Ens, W.; Standing, K. G. Collisional Damping Device for an Electrospray Ionization Time-of-flight Mass Spectrometer. J. Am. Soc. Mass Spectrom. 1998, 9(6), 569-579.

17. Qin, J.; Chait, B. T. Preferential Fragmentation of Protonated Gas-Phase Peptide Ions Adjacent to Acidic Amino Acid Residues. J. Am. Chem. Soc. 1995, 117, 5411-5412.

18. Qin, J.; Chait, B. T. Collision-induced Dissociation of Singly charged Peptide Ions in a Matrix-assisted Laser Desorption Ionization Ion Trap Mass Spectrometer. Int. J. Mass Spectrom. 1999, 190, 313-320.

19. Karas, M.; Bahr, U.; Strupat, K.; Hillenkamp, F. Matrix Dependence of Metastable Fragmentation of Glycoproteins in MALDI-TOF Mass Spectrometry. Anal. Chem. 1995, 6, 675-679.

20. Dawson, P. H. Quadrupole Mass Spectrometry and Its Applications. Elsevier Scientific Publishing Company: Amsterdam, 1976. 